\title{
Design and implement a self-managed computer network for electronic exams and sharing
}

Mokhaled N. A. Al-Hamadani, Ihab A. Sattam, Raid W. Daoud, Suhail N. Shehab, Haider A. Kamel Hawija Technical Institute (HTI), Northern Technical University (NTU), Iraq

\begin{tabular}{|c|c|}
\hline Article Info & ABSTRACT \\
\hline Article history: & \multirow{10}{*}{$\begin{array}{l}\text { To implement electronic exams and material resources sharing (which is } \\
\text { usually limited), an operating base is must be available for trading } \\
\text { information and correspondence management. In this paper, self-managed } \\
\text { computer networks, no server was designed. This network aims to share } \\
\text { information and correspondence management between the laboratory`s } \\
\text { computers. A specific software called Packet tracer used for designing and } \\
\text { simulating the network, also for choosing the right medium and install the IP } \\
\text { address so that a maximum of data flowing with minimum time and no data } \\
\text { loss can be achieved. A class-C IP address was selected since it is the famous } \\
\text { class and it doesn`t need any special equipment. Also it has a wide range of } \\
\text { of computers in case of expanding the network. We didn`t use a wireless } \\
\text { medium to protect the network from hacking. The network was based on an } \\
\text { Ethernet medium and a star-connection between the computers with an } \\
\text { average signal distributer. An excellent result was achieved after testing } \\
\text { the network with a low error percentage, as shown in the result section of } \\
\text { this paper. }\end{array}$} \\
\hline Received Jul 19, 2019 & \\
\hline Revised Dec 10, 2019 & \\
\hline Accepted Jan 18, 2020 & \\
\hline Keywords: & \\
\hline Data sharing & \\
\hline Host group & \\
\hline IP address & \\
\hline Network & \\
\hline Private host & \\
\hline
\end{tabular}

Copyright (C) 2020 Institute of Advanced Engineering and Science. All rights reserved.

\section{Corresponding Author:}

Raid W. Daoud, Department Electrical Techniques, Al-Sayada Q, Kirkuk, Iraq. Email: raid.daoud@gmail.com

\section{INTRODUCTION}

The computer networks need to several protocols and standards-setting to be in optimum work of operation. Network management is the process of managing resources of a network such as bandwidth, storage, CPU, etc. to enhance the performance of the network [1-3]. Bandwidth is a very essential, however expensive network resource that must be properly managed to provide the maximum required throughput expected by the network owners and users. Bandwidth can be defined in a variety of ways, counting on the context. In computing, bandwidth can be defined as the bit-rate of available or consumed information capacity measured in bits per second (bps). Bandwidth may also be called network bandwidth, data bandwidth, or digital bandwidth [4, 5]. In communication networks, Intermediate nodes (such as a router), and transmission bandwidth play the main role during data packets transmission [6-8]. As a result of that, computer communications networks consist of limited resources, which lead to increased competition existing between users on computer networks resources, and thus increasing the competitiveness as well as network resources become more limited [9, 10]. This leads to an overload on the network, leading to the deterioration of the efficiency of the network [11]. On a small, simple network, the problem is almost trivial, statically allocating routes and defining them by hand, but when dealing with a huge internetwork such as the Internet, this is not possible $[12,13]$. In the proposed network, the IP addresses are determined manually; there are simple numbers of the computer for data sharing, electronic exam issues, etc.

A user interacts with a service through a service access point. Note that as shown in the figure below, users interact with one service provider $[14,15]$. In practice, the service provider is distributed over 
several hosts, but these are implementation details that are not important at this stage. These interactions between a user and a service provider are expressed in [X200] by using primitives, as shown in Figure 1 [16]. These primitives are an abstract representation of the interactions between a user and a service provider. In practice, these interactions could be implemented as system calls, for example [17, 18].

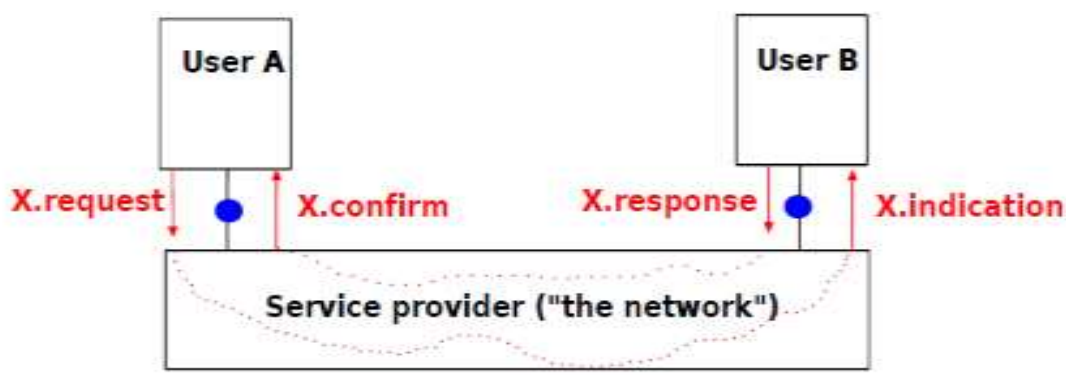

Figure 1. The four types of primitives

Simulation is becoming an increasingly popular method for network performance analysis. Software simulator is a valuable tool, especially for recent networks with complex architectures and topologies. A typical simulator can provide the programmer with the necessary information on how to control and manage the performance of a computer network. Functions and protocols square measure delineated either by the finite state machine, native programming code, or a combination of the two [19]. Computer networks have remarkably become essential in our present days. The majority of universities are using them as the best means of communication with all universities, scientific research institutes, and researchers around the globe. Besides, most universities have nowadays provided their entire curriculums online; thus, allowing students to have them without any difficulties [20,21]. Network simulators have developed since they first appeared as performance, management, and prediction tools. They usually are used as network management tools; that packet-level analysis is not ordinarily employed [22].

The main problem, will be solved by this proposed network, which is how to exchange secured data inside the computer lab, such as: student scores, questions, some standard answers, etc. In addition, packet transmission and transition tend to become easy accessible. Data security and privacy is the most affected key solution for the network [23]. Communication between networks are realised by unique IP and mobility of mobile stations is managed through protocols such as Mobile IP [12, 24]. Internet of Things (IoT) is a new generation of the latest technology which is envisioned as a future, this technology adds everyday devices with smart sensors, the device allows sender-receiver digital communication and is attached to a communication protocol that allows each device to connect with other devices [25].

\section{RESEARCH METHOD}

The network which has proposed in this paper contains conventional protocols and IP addresses as well as, rather than self-management for messages traveled through it. This is lead to get on the student mark or divide the questions on the computer lab by smart and live instructions on the network.

\subsection{System building}

The system`s structure was designed and built using Packet Tracer software [1] in which the Number of computers, the mediums.... etc. 20 machines were used. The medium was Ethernet cables, and a star-connection topographic was used. In case of protections the data lost and the correspondence safety, several things must be taken into consideration, which are:

a. Network Topology.

b. Protocol.

c. Class of IP-Address.

d. Subnet Address.

e. Medium.

All of the above must be determined according to a specific criterion; for example, the Protocol Depends on the medium. The medium depends on the distance for data transfer. The IP and the subnet Depend on the Number of Computers and the required security. The selection of the above leads to the high performance of the network by monitoring: 
a. Latency.

b. Throughput.

c. Packet; send and received "Losses."

d. Security.

\subsection{Type of network:}

A star- connection type is used for the proposed network since it is easy to connect the computers. The lab contains four columns of computers. Hub switch device is used to connect the computers at one point. The Hub switch was used as a feasibility study to decrease the number of wires used for the network, see Figure 2. Also the protocol used (CSMA/CD) has high accuracy and ensure that one computer is used in the network at a time.

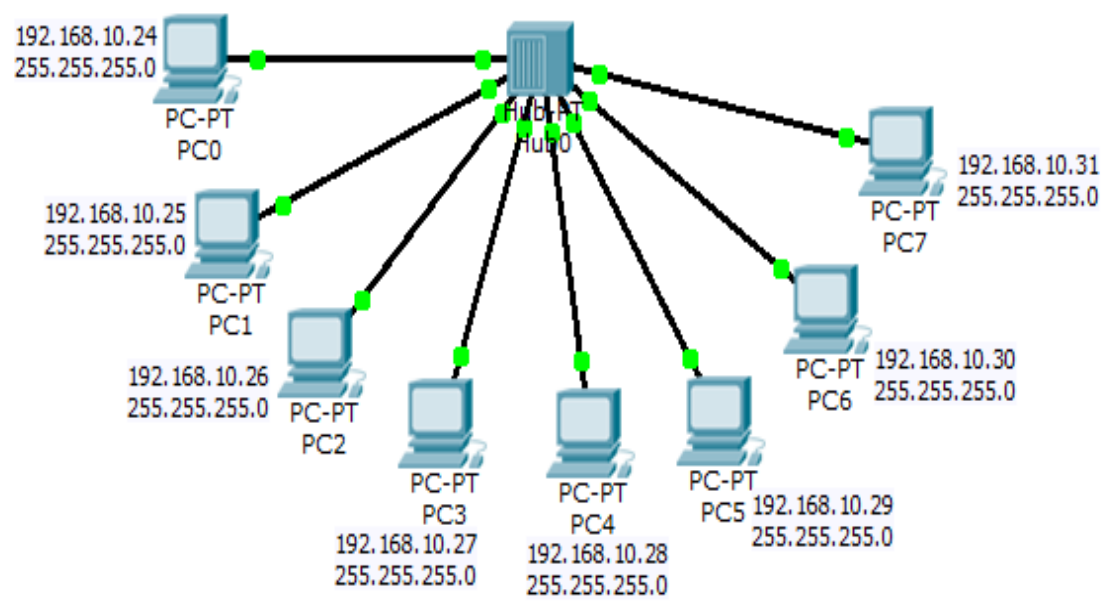

Figure 2. Network topology for the proposed work, star

\subsection{Network protocol}

It is many programs that control the managing of data flowing and allowing the computer to connect with another one in the network. As said in the previous section, the protocol used is (CSMA/CD) which ensures reservation of the network for one computer and prevents the colliding with more than one message sent from more than one computer. Another Protocol in Ethernet Network is (TCP), which is responsible for data transition between the sender and receiver and preparing the bands to adapt to the medium.

\subsection{IP address classes}

There are three types of classes for IP, which are as follows:
a. Class-A
1.0.0.1
126.255.255.254
b. Class-B
128.1.0.1
191.255 .255 .254
c. Class-C
192.0.0.1
223.255.255.254

Class-A is used in large companies that have (routers) with a large number of computers and servers. Class-B used in small organizations that have fewer computers and don't have (Routers). Class-C is the class chosen for the Network, used in small organizations and companies that have a few computers and network equIPment. The distributions of the addresses implemented according to a strategy that guarantees no repetition of the same address o more than one device. If so, the (CDMA/CD) protocol will isolate the devices with the same addresses from the network to proceed with the work of the network. As show in Figure 3, the distribution of the addresses starts with the server with address (192.168.10.10) than the computers than the other devices in the network such as a printer, scanner, etc. 


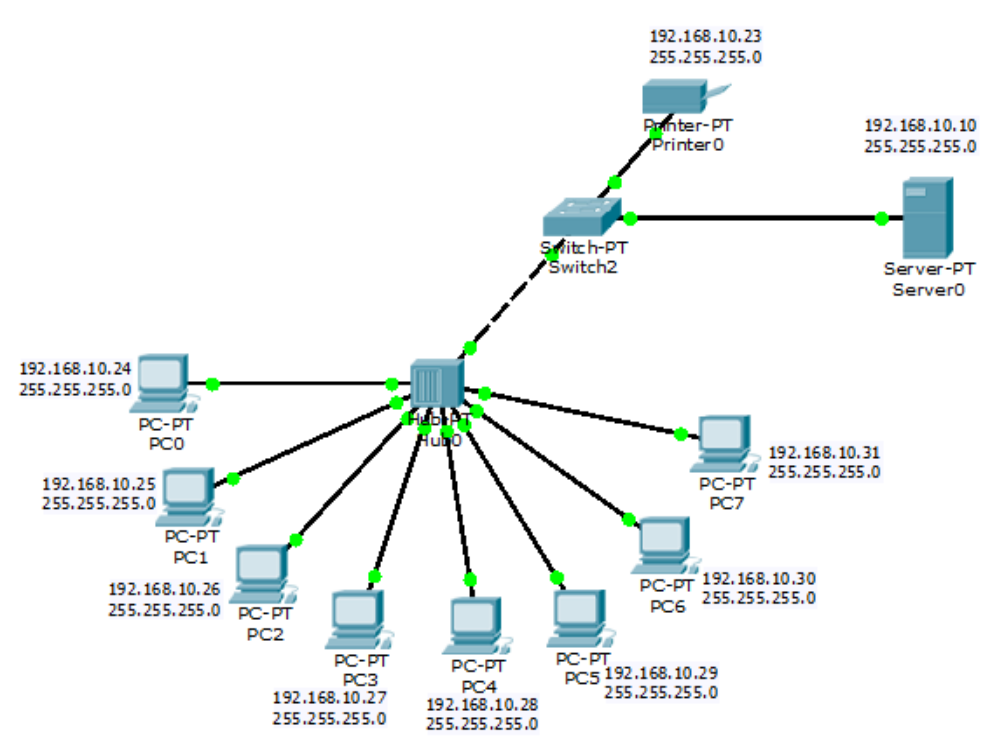

Figure 3. The IP address distribution on the proposed network

\subsection{Subnet address}

To make the network management easier and decrease error percentage, Network Fragmentation into two parts. Every class of IP has a certain band of (subnet), s for the class used in this paper is Class-C where the distribution of the IP is as follows:

\subsubsection{255.255.255.0}

This IP address makes the computers to work with a certain range, and no outside parts will be allowed to enter the network unless permission and the current IP are taken to include it in the network. As mentioned before, the purpose of this network is to increase the efficiency of the performance of the computer and keep the data confidential.

Also, it can be considered as a step forward for Smart Management. The computer Lab has exams for certain subjects in it, such as Microsoft Office, AutoCAD, and MATLAB. To collect the student's answer. In every computer network, several things must be considered and monitored to achieve high performance of the network.

\subsubsection{Latency}

It is the time consumed between the send and receive of the packet. The ideal time is (zero) second, but the medium has losses percentage in voltage and current coefficients. The real operation time doesn't exceed $1 \mathrm{~ms}$; see Figure 4. When a ping command has run between a given computer and the server on IP 192.168.10.10, to see the time is equal to ' 1 ' $\mathrm{ms}$ or ' 0 ' in some cases.

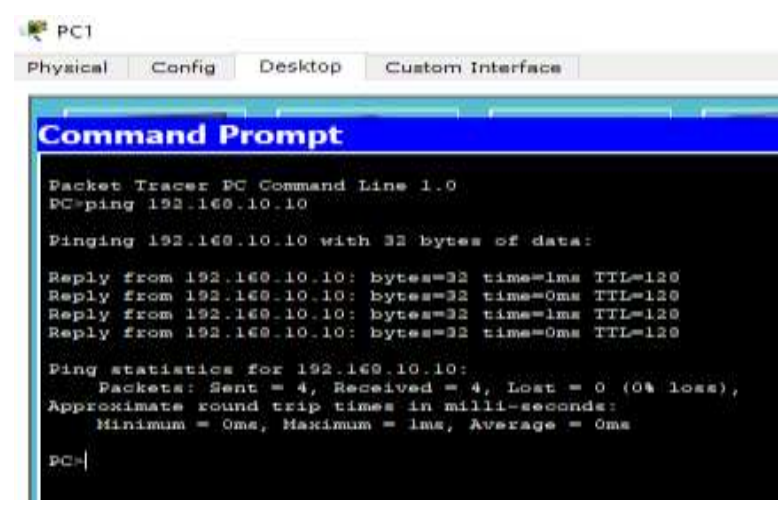

Figure 4. Simulation response for the proposed network 
The network was tested for both cases, the simulation and the actual case, and the error were less than $1 \mathrm{~ms}$. The network proved high efficiency for wide and medium bands (large data and simple data). Figure 5 shows the actual time in the network.

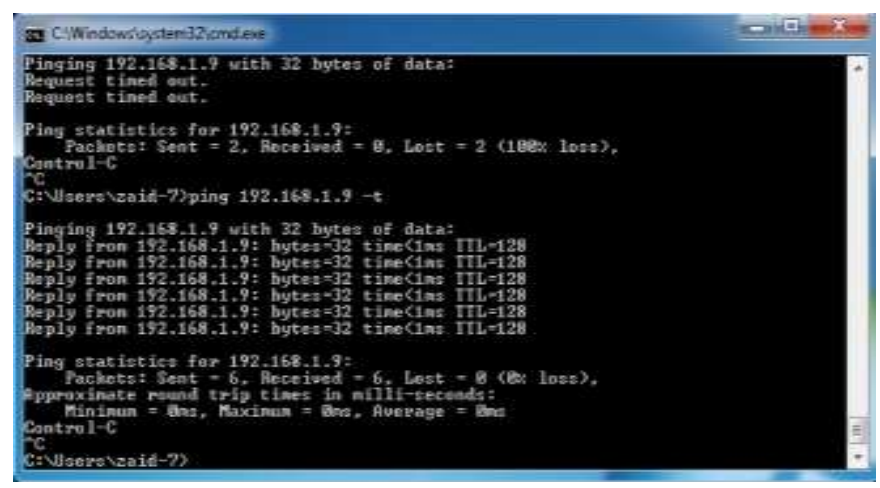

Figure 5. Real response on the physical network after installation

\subsubsection{Throughput}

In general terms, throughput is the rate of production or the rate at which something is processed. When used in the context of communication networks, such as Ethernet or packet radio, throughput or network throughput, is the rate of successful message delivery over a communication channel. The throughput of the proposed network reached the optimum case in the simulation and after installation. The packet tracer can send a message from one end to another and give the report, see Figure 6.

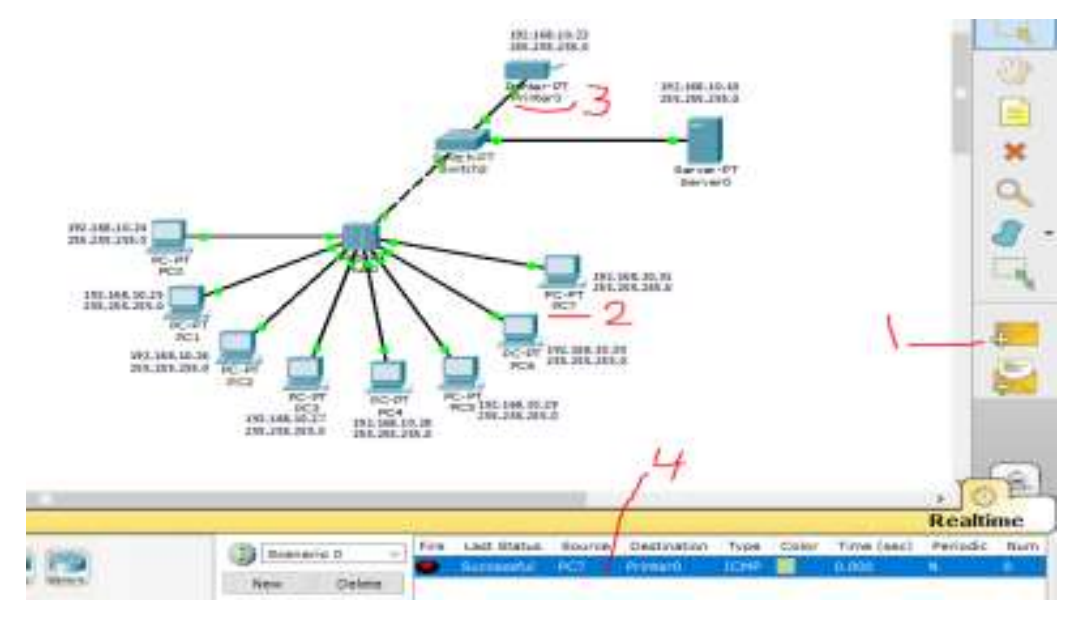

Figure 6. Throughput of the proposed network

\subsubsection{Packet; send and received "Losses."}

The computer network is produced for data and resource sharing, and the losses must be fully eliminated to reach the optimum word. As mentioned earlier, the losses are nearly zero in this network after the pre-test on the simulation program and after the hard installation of the network.

\subsubsection{Security}

A simple security manner is applied in this network, which is represented by using the static IP address for the computers and resources. In mind, there is a novel method for data security, which may be applied to the proposed network using artificial intelligence and will be future work. All the computers have a direct connection with the main Hub. The aim of the hub is to data transition between the network parts. Figure 7 shows the connection type of computers and how to choose the HUB position to collect a maximum number of computers with a short distance of wires. 


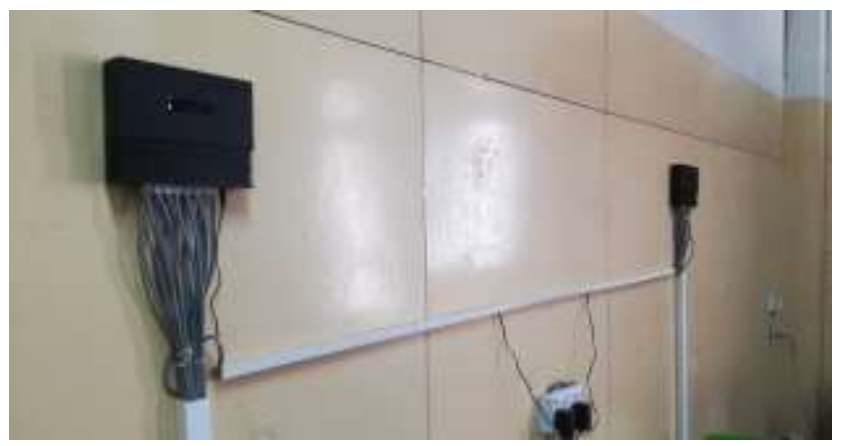

Figure 7. The physical connection of the network through the hub switch

\section{RESULTS AND ANALYSIS}

As mentioned earlier, the suggested network is a self-managed network where the data transition among the computers and between the computer and the printer is done without the interfering of the server. The network was designed based on static IP addresses. That means every computer has an IP that can be communicated with by another IP of another computer. This network aims to perform electronic exams. A certain application is used, and the test is given to the student, after finishing the test and collecting the answer, the result will be sent to a certain computer or the printer as the instructor desire. Figure 8 shows the data transfer mechanism between a computer and the printer. It can be noticed that the lab supervisor deals easily with the process. When the student finishes, the test lab supervisor will send the result directly to the network printer.

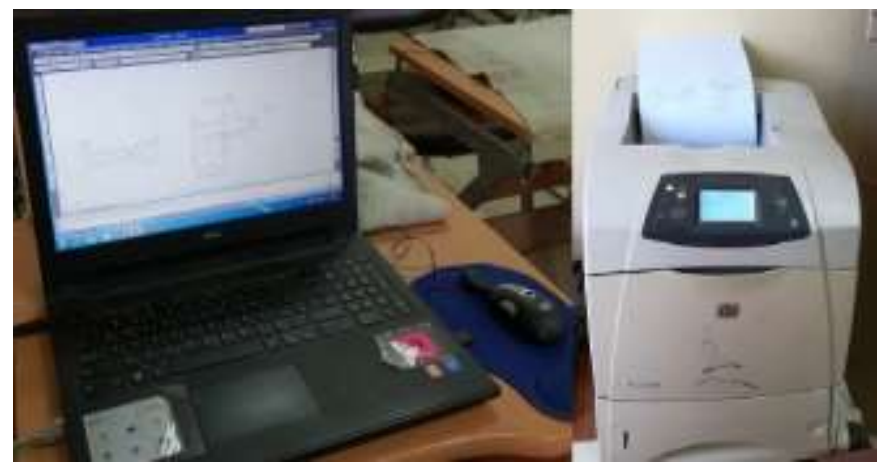

Figure 8. Laptop end with the network printer

\subsection{The Medium Selection}

For the suggested network, an Ethernet cable was selected due to technical and security reasons. For the technical side, the Ethernet cable can be easily installed since no complex equipment is required for the installation. Also, it is low cost and can transfer both power and data at the same time.

\subsection{Problems}

During the network installation, several problems appear. The distance between the computers and the connection among them is one of the problems, the position of the Hub and the distance between the hub and the computers have effects on the performance of the network. Various positions experimented. After several attempts, we decided to install two hubs to cover all the computers in the lab.

The coding pf computers is another problem we faced. Since this network aims to be a self-managed network, the work of this network should base on a certain protocol so that the network elements can communicate with each other at any time. The IP address used is a class-C due to a small number of computers, small space of the lab, and the network doesn't need a large amount of IPs. Also no extra equipment in the network such as routers or bridges. The first number was assigned to the main computer, which gives the commands to the network users, as shown in Figure 9. 


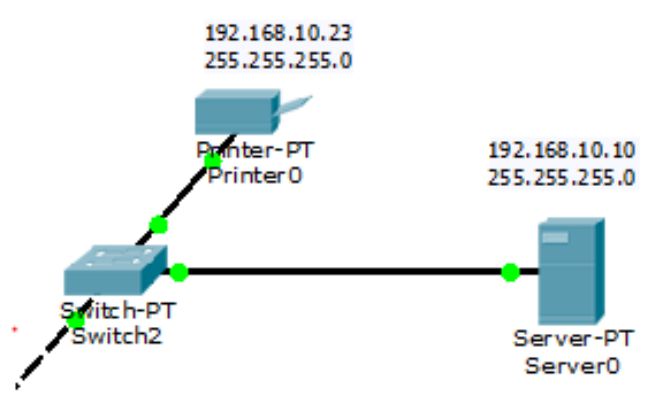

Figure 9. The IP address starting with sever at 192.168.10.10

Data management is implemented using the official protocol of the Local area Network (LAN), which is CSMA/CD. The protocol doesn't allow more than two devices to communicate at the same time, if such a problem appears, the third device will be isolated and delayed to a certain time until the communication between the first two devices is finished. Another problem we dealt with is the powering of the hub switch. Since it is an electrical device, it needs an AC electricity to power. The problem solved by applying an electricity system based on the solar cells to ensure the continuity of the network working at all times. An interesting fact about the protocol CSMA/CD, that allows two devices to communicate with each other, it doesn't mean that these two devices are the only devices that use the network, but every device in the network can communicate with each other without data intersections.

For example, when the user used the printer from one of the computers, any other computer won't be allowed to use that printer until the first computer finished. In case of such a problem, the network manager using a certain device will delay the message by containing her with a buffer source and sent it at a certain time. The delay Time due to data conflicts, which is forcing the network manager to delay it, was recorded. The readings were so close to the simulation readings.

Finally, one of the biggest problems that delayed the work of the network is the lack of an active hub due to financial issues. This problem causes some computers to lose their connection with the network. Despite that, the network is self-managed, but it basically depends on the speed response of the hub, which was the passive type, which leads to slow at work and weak managing of the network. Figure 10 shows how some computers lose their connections with the network. This problem is solved by defining the homegroup for the network to force the work on a certain number of users and define the CSMA/CD protocol in a specific manner, see Figure 11. The homegroup process is needed to some fixed operation before the installation, such as: (IP address, online hub switch, more than one PC). By creating the group of operation for the proposed computer network, all the terminals are communicating with the network devices in a proper manner without any delay and loss of connection problem. The "conflict IP address" message is usually appears when the network operates in a given mode by supervision a passive router. The network which is used the internet, wireless connection or either ethernet connection, rarely face the conflict IP address problem because the global DHCP protocol is the super supervisor for the addresses. However, the network, for the small purposes and printer sharing, must have an active router to overcome the IP addresses subject.

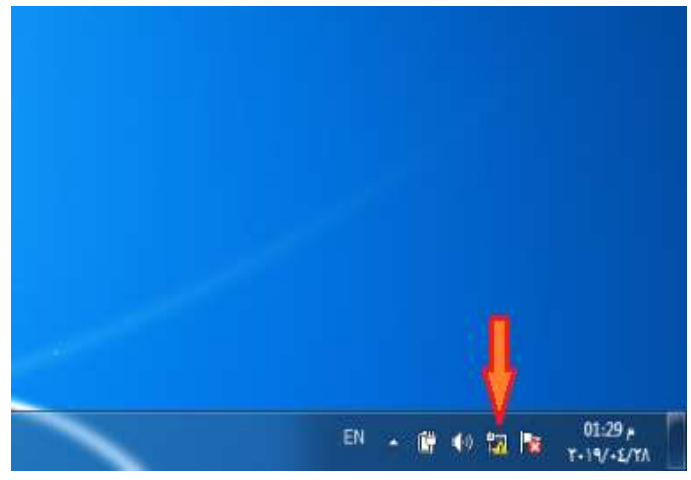

Figure 10. Effect of the passive hub on the network

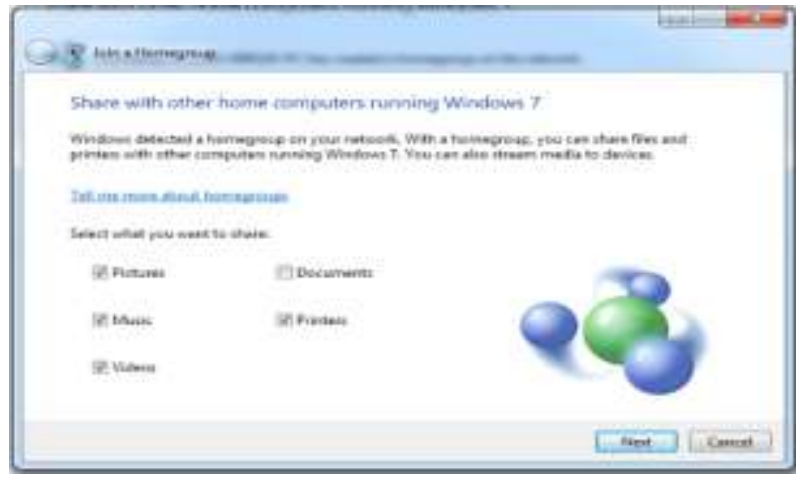

Figure 11. Homegroup defining for the network 
The main results of the proposed network are the minimized latency and nothing data loss during the simulation and real network operation. Figures 12 and 13 shows the response of the network and the received message case. The loss data problem may be occurred during the bad network media or not supported protocol is installed. In this proposed network, standard network protocols are used, rather than an innovative static IP has used to eliminate the conflict IP address problem in addition to a more secure data connection. In the computer lab, especially those determined for exams and private data sharing, the data exchange must be transferred through a secured channel, and this is done by select the active hup switches and static IP address connection method.

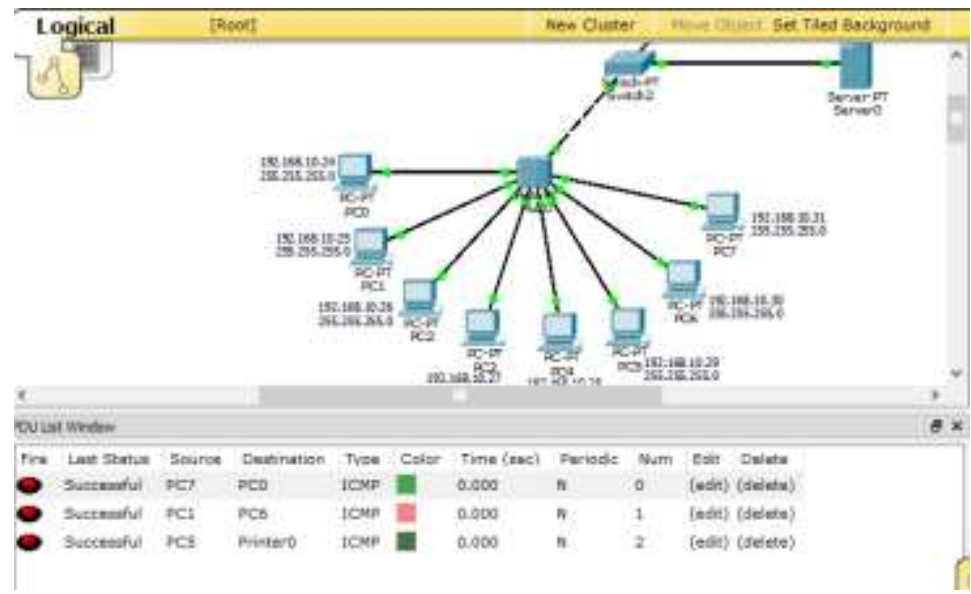

Figure 12. Message transmission between the network elements

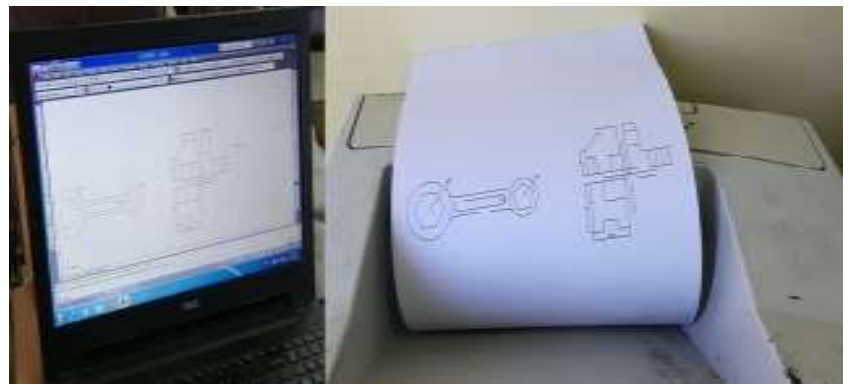

Figure 13. No losses on the real network after installation

\section{CONCLUSION}

The self-management network, which is proposed in this work, has several benefits: 1) The computer lab transferred to smart one by adding an auto print for the achieved exam without the need to save it on a given device; this is a new manner; 2) Packet tracer simplifies the work by implementing and test the network before the hard installation; 3) The static IP address serves the work; each terminal knows his address and no need to add IP provider "router," which may produce additional problems, 4) The CSMA/CD manages the network and gives an optimal response; this is monitored by packet tracer.

\section{ACKNOWLEDGEMENTS}

Thanks to the administrator of the computer lab which is prepared all the facilities to build and manage the computers in the proposed manner.

\section{REFERENCES}

[1] Ammar. T. Namel, et al., "Bandwidth Utilization Prediction in LAN Network Using Time Series Modeling," Iraqi Journal of computers, communication, and control \& systems engineering, vol. 19, no. 2, pp. 78-89, 2019. 
[2] C. C. Praude, "Computer Art and Actor-Network Theory: Actants and Intersubjective Associations in Scene," Leonardo (Johns Hopkins University Press), vol. 51, no. 5, pp. 529-529, 2018.

[3] R. W. Daoud and W. S. Hassan, "Neural Network Based of a New User IP Address Determination and Address Conflict Elimination," Diyala Journal of Engineering Sciences, vol. 12, no. 03, pp. 15-21, 2019.

[4] S. Kim, "Heterogeneous network bandwidth management scheme based on the princIPal-agent game model," Wireless Networks, vol. 23, pp. 279-287, 2017.

[5] V. der H. Jeroen, et al., "Updated Taxonomy for the Network and Service Management Research Field," Journal of Network and Systems Management, vol. 26, no. 3, 2018.

[6] R. Benjamin J., et al., "Sequence Aggregation Rules for Anomaly Detection in Computer Network Traffic," arXiv, 2018.

[7] W. Villota, et al., "On the Feasibility of Using Hierarchical Task Networks and Network Functions Virtualization for Managing Software-Defined Networks," IEEE Access.

[8] H. Xua, et al., "Reducing controller response time with hybrid routing in software defined networks," Computer Networks (sciencedirect), 2019.

[9] K. A. Nassar and A. A. Abdullah, "Fuzzy RED to Reduce Packet Loss in Computer network," Journal of AlQadisiyah for Computer Science and Mathematics, vol. 8, no. 1, pp. 107-114, 2016.

[10] R. W. Daoud and R. H. A. Al-Mallah, "Design and Simulate an Attenuator for Multi Types Optical Fiber Using Neural Networks," International Journal of Enhanced Research in Science, Technology \& Engineering, vol. 8, no. 5, pp. 19-26, 2019.

[11] J. Zue, et al., "Fuzzy Logic Aided Dynamic Source Routing in Cross-Layer Operation Assisted Ad Hoc Networks," Southampton University, United Kingdom, 2010.

[12] A. Dana and N. Salehi, "Congestion Aware Routing Algorithm for Mesh Network-on-ChIP Platform," Indian Journal of Science and Technology, vol. 5, no. 6, 2012.

[13] Z. Shifeng, et al., "Improved Selective Refinement Network for Face Detection," arXiv, 2019.

[14] S. C. Tharaka, et al., "High Security Firewall: Prevent Unauthorized Access Using Firewall Technologies," International Journal of Scientific and Research Publications, vol. 6, no. 4, pp. 504-508, 2016.

[15] H. Kaur, et al., "IP Address Blocking System," International Journal on Computer Science and Engineering (IJCSE), vol. 8, no. 4, pp. 133-139, 2016.

[16] Y. Qiua, et al., "Experimental evaluation of LXC container migration for cloudlets using multipath TCP," Computer Networks (sciencedirect), 2019.

[17] O. Bonaventure, "Computer Networking: Principles, Protocols, and Practice," The Saylor Foundation, 2011.

[18] R. Kaur and A. Kaur, "Safeguard of Security: Firewalls," International Journal of Innovative Research in Computer and Communication Engineering, vol. 5, no. 3, 2017.

[19] M. B. Abdullah and A. I. A. Jabbar, "Towards the Improvement of the Computer Network of Mosul University Using (OPNET) Software," AL Rafdain Engineering Journal, vol. 15, no. 1, pp. 15-26, 2007.

[20] J. Heiddemann and K. Mills, "Expanding Confidence in Network Simulations," IEEE Network Magazine, vol. 15, no. 5, 2001.

[21] K. Pawlikowski, et al., "The Credibility of Simulation Studies of Telecommunication Networks," IEEE Communications Magazine, vol. 40, no. 6, 2002.

[22] M. A. Hammoudi, "Building Model for the University of Mosul Computer Network Using OPNET Simulator," Tikrit Journal of Eng. Sciences, vol. 18, no. 2, pp. 34-44, Jun 2011.

[23] I. Ahmed, "A brief review: security issues in cloud computing and their solutions," TELKOMNIKA Telecommunication Computing Electronics and Control, vol. 17, no. 6, pp. 2812-2817, Dec 2019.

[24] R. Shankar and P. Dananjayan, "Quality of Service in bandwidth adapted hybrid UMTS/WLAN interworking network," TELKOMNIKA Telecommunication Computing Electronics and Control, vol. 17, no. 6, pp. 2803-2811, Dec 2019.

[25] A. Mayub, et al., "Implementation smart home using the internet of things," TELKOMNIKA Telecommunication Computing Electronics and Control, vol. 17, no. 6, pp. 3126-3136, Dec 2019.

\section{BIOGRAPHIES OF AUTHORS}

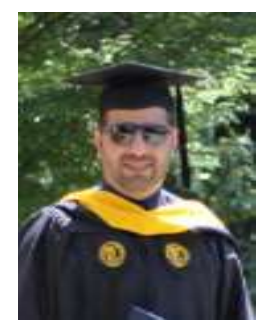

Mokhaled N. A. Al-Hamadani Bachelor of Technology in Computer Science-Computer Science Dept./College of Science/ University of Kirkuk, Iraq.Master of Science in Computer Science/Computer Science Dept./ College of Arts and Sciences/ The University of North Carolina at Greensboro. Assistant Lecturer at Electronic Techniques Dept./ Al-Hawija Technical Institute/ Northern Technical University. 


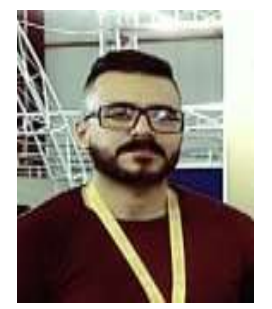

Ihab Abdulrahman Satam B.Sc Mechatronics-Engineering college-University of Mosul-Iraq. M.Sc Mechatronics-Alkhwarizmi Engineering college- University of Baghdad-Iraq. Assistant lecturer - Northern Technical University- Iraq. Research interest. Robotics - Neural Networkcontrol - Autopilot

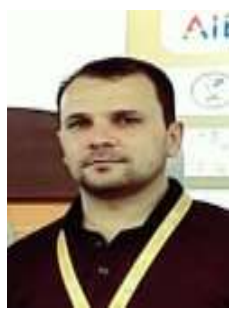

Raid W. Daoud, B.Sc in Techniques of computer engineering college, Iraq, 2003, and the M.Sc from the same college in 2008 . He has more than one paper published in different computer subjects, such as control and optical fiber. 